WellBeing International

WBI Studies Repository

3-2012

\title{
The Ban on the Use of Chimpanzees in Biomedical Research and Testing in the UK Should Be Made Permanent and Legally Binding
}

Michelle Thew

British Union for the Abolition of Vivisection

Jarrod Bailey

British Union for the Abolition of Vivisection

Michael Balls

Fund for the Replacement of Animals in Medical Experiments

Michelle Hudson

Fund for the Replacement of Animals in Medical Experiments

Follow this and additional works at: https://www.wellbeingintlstudiesrepository.org/acwp_lab

Part of the Animal Experimentation and Research Commons, Animal Studies Commons, and the Other Law Commons

\section{Recommended Citation}

Thew, M., Bailey, J., Balls, M., \& Hudson, M. (2012). The ban on the use of chimpanzees in biomedical research and testing in the UK should be made permanent and legally binding.

This material is brought to you for free and open access by WellBeing International. It has been accepted for inclusion by an authorized administrator of the WBI Studies Repository. For more information, please contact wbisr-info@wellbeingintl.org.

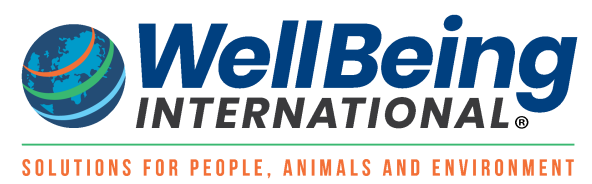




\title{
Submission
}

\section{The Ban on the Use of Chimpanzees in Biomedical Research and Testing in the UK Should Be Made Permanent and Legally Binding}

\author{
A joint submission to the Coalition Government \\ by the BUAV and FRAME
}

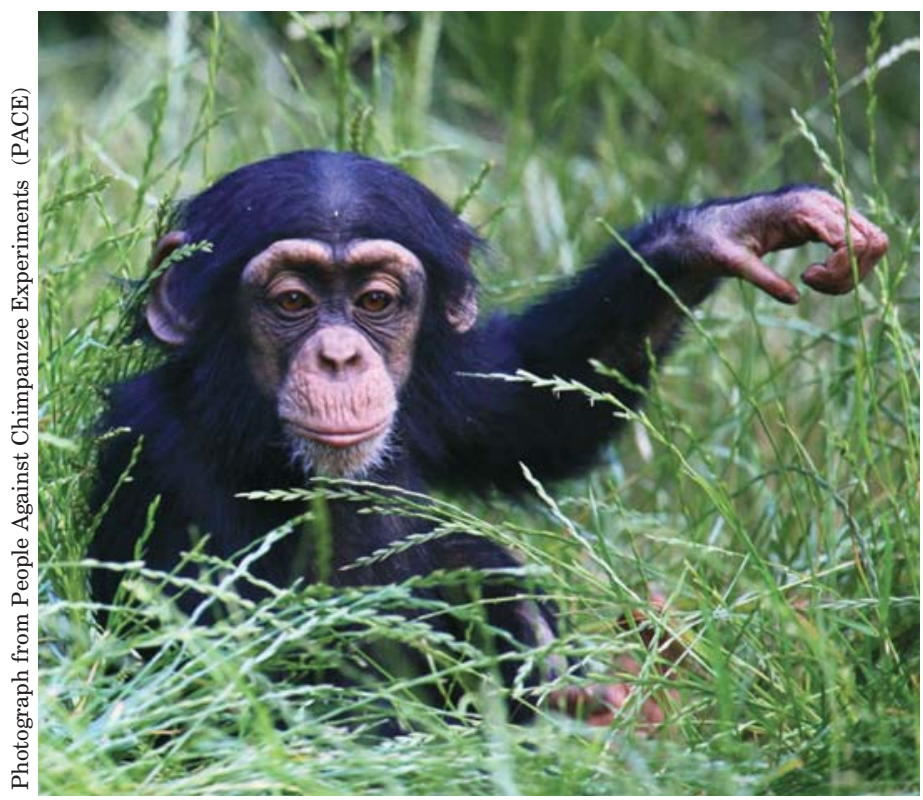

The BUAV and FRAME jointly urge the Government to put this issue beyond doubt, by making the ban on experiments on Great Apes permanent and legally binding, as would be permitted by Article 2.1 of the Directive. This would be entirely justifiable on ethical, scientific, logistic and economic grounds, as outlined in the supporting information below. Eliminating the possibility of Great Ape experiments would be accepted, indeed welcomed, by both the scientific and the animal welfare communities.

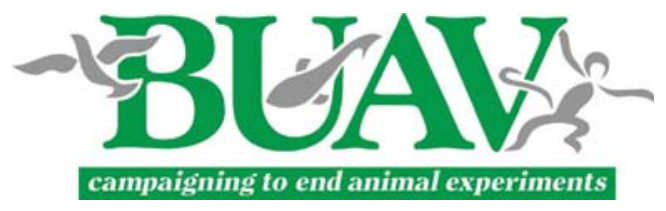

Michelle Thew

Jarrod Bailey

for the British Union for the Abolition

of Vivisection (BUAV)

16a Crane Grove

London N7 8NN, UK

e-mail: michelle.thew@buav.org
The Coalition Government is currently considering how to transpose Directive $2010 / 63 / E U$ on animal experimentation into UK law. The Directive bans the use of Great Apes in laboratories, but EU Member States can seek (now or, more likely, at some time in the future) a derogation from the Commission to permit such use, where this is considered essential for the preservation of the species in question or in relation to an unexpected outbreak of a life-threatening or debilitating clinical condition in human beings. Currently, the policy of the Government is not to approve any experiments on Great Apes, but it is possible that, at some time in the future, researchers might ask the then Government to seek permission via the derogation clause.
Michael Balls

Michelle Hudson

for Fund for the Replacement of Animals

in Medical Experiments (FRAME)

96-98 North Sherwood Street

Nottingham NG1 4EE, UK

e-mail:michael.balls@btopenworld.com

February 2012 


\section{Supporting Information}

\section{Public opinion and support for a ban}

At present, chimpanzees are used in research only in the USA. Many other countries have banned, or at least severely restricted, research and testing involving chimpanzees, including the UK, which banned the licensing of Great Ape research in 1997. There is great public opposition to such use: the American public are twice as likely to support a ban on chimpanzee research as to oppose it, according to a public opinion survey carried out in 2005 (1). In 2009 in the UK, 81\% of the public agreed that experiments causing pain or suffering to any non-human primates should be banned (2).

\section{Ethical Considerations}

In the UK, and now throughout the EU, the moral justification for conducting animal experiments is assessed via a cost/benefit analysis. The costs to the animal in terms of suffering, pain and distress must be outweighed by the potential benefits of the scientific outcomes to humans, animals or the environment for the proposed work to be permitted. The scientific information presented here provides strong evidence that the costs always far outweigh the benefits in the case of chimpanzee experiments, since the psychological and physical harms to these animals profoundly exceed the minimal if any benefits accrued from the research. In addition to these examples the endangered status of all Great Apes must be taken in to account, with the threat of extinction being an additional cost that must be considered.

\section{Psychological harm}

Recent papers published in peer-review psychological journals demonstrate how chimpanzees used in research suffer from severe and lasting emotional trauma:

Humanity, Reason, and Justice: Law of Psychiatric Injury and the Bioethics of Great Ape Wellbeing (3) describes the application of American Psychiatric Association criteria, codified for assessing human mental states, to the evaluation of Great Ape psychological well-being. This nullifies scientific arguments that defend a double ethical and legal standard for how individual chimpanzees and human beings should be treated, as it acknowledges psychiatric damage in Great Ape biomedical research subjects.

Building an Inner Sanctuary: Complex PTSD in Chimpanzees (4) is a clinical case study of the symptoms of Posttraumatic Stress Disorder (PTSD) shown by two chimpanzees used for several years in biomedical research, before reaching sanctuary. The findings show that chimpanzees in laboratories suffer from PTSD, just as humans do when they survive traumatic events. This paper's findings confirm what is known from other studies, i.e. that it is virtually impossible to use chimpanzees in biomedical research without causing them extreme and lifelong psychological suffering.

Signs of Mood and Anxiety Disorders in Chimpanzees (5) describes how chimpanzees display behavioural clusters similar to PTSD and depression in their key diagnostic criteria, underscoring the importance of ethical considerations with regard to the use of chimpanzees in experimentation and in other captive settings.

Developmental Context Effects on Bicultural Post-Trauma Self Repair in Chimpanzees (6) examines the case histories of three chimpanzees used in research before being rescued into sanctuary. The findings show that chimpanzees separated from their mothers and cross-fostered by humans, not only suffer from being used in research, but also have the compounded trauma of an identity crisis resulting in compromised ability to socialise with other chimpanzees. This study provides further evidence of how many chimpanzees never fully recover from laboratory confinement and use, but continue to suffer from psychological problems, even in sanctuary.

\section{Physical harm}

Laboratory life also causes physical harm to chimpanzees (7). Individual chimpanzees are used repeatedly to obtain blood, serum, tissue, and other biological specimens, or in multiple protocols. Since captive chimpanzees can live an average of 55 years, this can mean decades of multiple experiments, knockdowns and other procedures. Centralisation and "recycling" chimpanzees into multiple research protocols adds to the suffering of these highly sensitive and cognisant individuals. In hepatitis C research, for example, currently the major use of research chimpanzees, infected chimpanzees live in isolation in sterile biocontainment, where they are subjected to frequent blood withdrawal and other invasive procedures. In an experiment on hepatitis B in 2004, for example, each of three chimpanzees was anaesthetised with a dart gun 29 times dur- 
ing the 6.5-month duration of the experiment, in order to be infected, to be injected with a test therapeutic agent, and to undergo needle liver biopsies (8). It can take up to five darts to successfully knock down an adult chimp. Some chimpanzees in laboratories have been knocked down more than 200 times, and have had more than 130 liver biopsies performed $(9,10)$.

In research facilities, chimpanzees typically live in small groups or pairs, in cramped concrete pens or indoor/outdoor runs, or alone in a $5^{\prime} \times 5^{\prime} \times 7^{\prime}$ cage. In such cages, large male chimpanzees can barely stretch out vertically; much less perform natural behaviours such as swinging from one hold to another. Some chimpanzees have lived their entire lives in these cages, with no outdoor access or windows for fresh air or sunlight. Others are housed in these small cages while being used in a protocol, since it gives easier 'access' to them. This confinement can go on for decades.

Chimpanzees often endure decades of endless boredom, with nowhere to go and nothing to do. They must cope with the constant stressful sounds of other traumatised chimpanzees, as well as the constant laboratory noises of cage banging and rattling, lock clanging, and cleaning hose roaring. Because chimpanzees are several times stronger than humans, they usually must be anaesthetised for even minor procedures, such as injections or blood withdrawal. A knockdown typically involves one or more workers, who approach the chimpanzee through the bars with a dart gun loaded with anaesthetic. It often requires several darts to knock down a chimpanzee as the animal screams and thrashes around in a futile attempt to avoid being shot, while darts have been known to hit them in the face or other sensitive areas. It is so terrifying that they typically lose control of their bladders and bowels during this procedure. They often know when a knockdown is about to occur, because their food and water is withheld, leaving them in anxious anticipation.

Chimpanzees used in laboratories endure frequent and considerable pain. Reviews of records from closed laboratories have revealed egregious research procedures, such as back surgery (i.e. removing discs or purposely causing back problems), that were performed without administering any post-surgical pain medication. In some laboratories, chimpanzees were not given pain relievers after invasive procedures such as liver punch or wedge biopsies.

\section{Scientific Ineffectiveness}

The scientific community itself is questioning the benefits which can be obtained through the use of chimpanzees, because of poor results, enormous costs, ethical concerns, and growing public opposition. Five US laboratories have either closed or released their chimpanzees to sanctuary. Active protocols using chimpanzees are few, while chimpanzees are warehoused in US laboratories (an estimated $80-90 \%$ of chimpanzees housed in US laboratories are not in protocols at any one time). Recently published studies demonstrate the scientific flaws and dangers of chimpanzee research, showing that it is not effective, efficient, or necessary for human health and well-being:

Chimpanzee Research: An Examination of its Contribution to Biomedical Knowledge and Efficacy in Combating Human Diseases (11) was based on a citation analysis of 95 randomly selected papers published between 1995 and 2004. The results indicate that the majority of chimpanzee research published (in addition to research not submitted or not accepted for publication) is never subsequently cited in human medical studies. On the rare occasion that they were cited, it was clear that the chimpanzee experiments had contributed little, if anything, to the outcomes described in papers reporting an advance in human clinical practice. In essence, the few chimpanzee research studies that have been published have had a limited, if any, impact on human health or medical advances.

An Assessment of the Role of Chimpanzees in AIDS Vaccine Research (12) demonstrates the failure of chimpanzee research in leading to a human AIDS vaccine. To date, 85 different vaccines have been shown to be safe and efficacious in chimpanzees and monkeys. However, in 197 clinical trials, protection and/or significant therapeutic effects have not been demonstrated by any of the vaccines in humans. The HIV infection of chimpanzees does not result in an AIDS-like illness comparable with that which occurs in humans. The use of chimpanzees in AIDS research has declined to the point of being hardly existent. There is no justification for a return to the use of chimpanzees in AIDS research or vaccine development.

An Examination of Chimpanzee Use in Human Cancer Research (13) analysed the use of chimpanzees in human cancer research from 1968 to 2008. It was found that chimpanzees, our closest genetic relatives, have contributed little to combating cancers, and not only cost time and human resources, but wasted research funding. Chimpanzee tumours are extremely rare, and are biologically different from human cancers. Major differences at the cellular level make chimpanzees a poor model for humans in relation to cancer, despite our 
overall genetic similarity. Chimpanzees are not essential (or even useful) in the development of human cancer therapies, and cancer research would not suffer if chimpanzee use came to an end.

\section{An Assessment of Chimpanzee Use in Hepatitis C Research, Past, Present and Future: 1. Validity of the} Chimpanzee Model (14), and 2. Alternative Replacement Methods (15) demonstrate: that claims of the historic usefulness of chimpanzees in hepatitis $C$ research are exaggerated; that chimpanzee use has persisted out of convention rather than scientific merit or necessity, despite superior alternatives; and that assertions concerning the critical role of chimpanzees in future hepatitis $C$ research are scientifically unjustifiable. Countless methods not involving chimpanzees have greatly informed hepatitis $C$ research, including: the culture of the hepatitis $C$ virus in human cells; studies on patients and human tissues; and the use of computer models. These approaches facilitate thorough investigation of the complete lifecycle of the hepatitis $C$ virus and the discovery and development of therapeutic agents/vaccines in a human context. The two assessments led to the conclusion that prohibiting chimpanzee hepatitis $C$ research would accelerate progress against the disease, and that unsubstantiated claims of the necessity of chimpanzee hepatitis $C$ research should not dissuade a public policy change regarding the confinement and use of chimpanzees in US laboratories.

Furthermore, chimpanzees have been used in only a relatively small number of research projects. Hepatitis $\mathrm{C}$ research not based on chimpanzees has increased 80 -fold over the last 20 years. By contrast, hepatitis $\mathrm{C}$ research involving chimpanzees has declined by almost $70 \%$, and is at a historic low. GlaxoSmithKline have a major interest in developing hepatitis C therapies, but decided in 2008 (16) that they had no need to use chimpanzees in their research and development.

Lessons from Chimpanzee-based Research on Human Disease: The Implications of Genetic Differences (17) shows that the genetic similarity between humans and chimpanzees is actually about $94 \%$, not $98-99 \%$, as is often claimed. Interspecies differences in genes and gene expression affect, for example: immune system function, autoimmune diseases such as lupus and rheumatoid arthritis; cancer initiation and progression; susceptibility to HIV and other viral infections and their related pathology; schizophrenia and other cognitive disorders; migraine; and the neurodegeneration linked to Alzheimer's, Parkinson's and Huntington's diseases. Such differences in gene expression occur throughout the body. Even small variations can result in significant effects such as reducing jaw muscles and changing brain development in humans. Only slight changes in a single gene (FOXP2) allowed humans to acquire unique language abilities.

\section{Logistic Considerations}

One of the main arguments for a derogation to permit the use of chimpanzees in laboratories, even if solely in extreme circumstances, is the possibility of a new threat to human health through the emergence of a new or more virulent pathogenic agent, or via bioterrorism. However, at a conference in the USA on chimpanzee research in 2011 (18), an expert on public health indicated that chimpanzees were 'probably not' critical to the health security of the United States, citing the many years it takes, for example, to develop a vaccine, and that any health scare would be long over before anything could be developed. They also noted the problem of the maintenance costs for chimpanzee colonies, even if they were deemed useful, at approximately half a million dollars per chimpanzee for lifetime care. The Director of the Office of Biodefense Research Affairs at the National Institutes of Health (NIH) stated that the chimpanzee offers 'no advantage over other nonhuman primates for product development for biodefense', citing current existing protection against many agents, such as smallpox, botulism, and bubonic plague. The Director of the National Biodefense Analysis and Countermeasures Center stated that he was 'not aware of any historical or current use of chimpanzees in the USA in biodefense for the Department of Defense', and that he did not anticipate any future speculative need.

At present, no chimpanzees are available in the UK for use in laboratories. Even if such use were ethically acceptable and scientifically justifiable, it would take several years to provide sufficient animals for use in meaningful experiments, even if the need for it could be foreseen in advance. This, coupled with the endangered status of all Great Apes, makes their use logistically impossible and, as the following section indicates, unrealistic for economic reasons.

\section{Economic Considerations}

If the use of chimpanzees in UK laboratories were ever to be considered, the formidable economic costs involved would have to be addressed. Figures from American institutions show that while quality of care (diversity of housing, access to outdoor areas, enrichment, diets of fresh fruit and vegetables) in reputable sanctuaries is superior to laboratory care, it costs more to house and maintain a chimpanzee in a laboratory than in a sanctuary. For example: the MD Anderson Cancer Center received $\$ 53$ and Alamogordo Primate 
Facility received $\$ 67$ per day per chimpanzee from the NIH/NCRR to house and maintain chimpanzees, whereas superior sanctuary care costs $\$ 38-\$ 40$ per day. In addition, 'indirect costs' that have little if anything to do with the direct care of the chimpanzees, such as research facility operation, maintenance and, general administration and expenses, add significantly to the costs of any proposed chimpanzee research. In the US: New Iberia Research Center received more than \$1 million for about 325 chimpanzees, $44 \%$ of which was for indirect costs; MD Anderson Cancer Center received $\$ 3.6$ million for about 200 chimpanzees, including $50 \%$ for indirect costs; and Yerkes National Primate Research Center received \$2.1 million for about 75 chimpanzees, including $37 \%$ for indirect costs. It has been estimated that the US federal government would save more than $\$ 8$ million dollars a year by retiring all federally owned and supported chimpanzees to sanctuaries. At its greatest this equates to approximately $\$ 28,000$ (approximately $f 18,000$ ) per chimpanzee per year. This does not include the costs of building or adapting facilities to make them suitable for housing the animals and for containing the biological agents that they would be subjected to, of acquiring and transporting the animals, or of the increased security which institutions would inevitably have to introduce, which would run into many millions, if not billions, of pounds.

\section{Conclusion}

In the UK, logistic and economic restrictions mean that experiments on chimpanzees could not be the basis for a rapid response to an emergency of any kind, even if a colony held in reserve could be provided and maintained. The costs to these animals are immense, and whether there are real benefits to be gained from research on them is highly questionable. The way forward in relation to all human diseases should be to take advantage of the new and advanced technologies which can be directly and ethically applied to humans, or which use cells and tissues derived from humans. The case for putting into place a permanent, legal ban on the use of Great Apes in experiments in the UK is overwhelming and unanswerable. Therefore, we urge the Government to take the opportunity offered by the transposition of the new EU directive into UK law, by adding such a ban to the revised Animals (Scientific Procedures) Act.

\section{References}

1. Project R \& R (2006). Public Opinion. Boston, MA, USA: NEAVS. Available at: http://www.releasechimps.org/ mission/end-chimpanzee-research/public-opinion/ (Accessed 23.1.12).

2. ECEAE (2009). Public Opinion. London, UK: European Coalition to End Animal Experiments. Available at: http://www.eceae.org/en/what-we-do/campaigns/12-million-reasons/public-opinion (Accessed 23.1.12).

3. Capaldo, T. \& Bradshaw, G.A. (2012). Humanity, Reason, and Justice: Law of Psychiatric Injury and the Bioethics of Great Ape Wellbeing. Policy Paper, 54pp. Ann Arbor, MI, USA: Animals \& Society Institute.

4. Bradshaw, G.A., Capaldo, T., Lindner, L. \& Grow, G. (2008). Building an inner sanctuary: Complex PTSD in chimpanzees. Journal of Trauma and Dissociation 9, 9-34.

5. Ferdowsian, H.R., Debra, L., Durham, D.L., Kimwele, C., Kranendonk, G., Otali, E., Akugizibwe, T., Mulcahy, J.B., Ajarova, L. \& Johnson, C.M. (2011). Signs of mood and anxiety disorders in chimpanzees. PLoS One 6, e19855.

6. Bradshaw, G.A., Capaldo, T., Lindner, L. \& Grow, G. (2009). Developmental context effects on bicultural posttrauma self repair in chimpanzees. Developmental Psychology 45, 1376-1388.

7. Project R \& R (2009). Chimpanzee Lab Life. Boston, MA, USA: NEAVS. Available at: http://www.releasechimps. org/harm-suffering/chimpanzee-lab-life/ (Accessed 23.1.12).

8. Wieland, S., Thimme, R., Purcell, R.H. \& Chisari, F.V. (2004). Genomic analysis of the host response to hepatitis B virus infection. Proceedings of the National Academy of Sciences of the USA 101, 6669-6674.

9. Project R \& R (2009). Hepatitis Detour. Boston, MA, USA: NEAVS. Available at: http://www.releasechimps.org/ harm-suffering/research-current/hepatitis-detour (Accessed 11.2.12).

10. Fauna Foundation (undated). Billy Jo. Chambly, Québec, Canada: Fauna Foundation. Available at: http://www. faunafoundation.org/html/billyjoprofile.html (Accessed 11.2.12).

11. Bailey, J., Balcombe, J. \& Capaldo, T. (2001). Chimpanzee Research: An Examination of Its Contribution to Biomedical Knowledge and Efficacy in Combating Human Diseases. 47pp. Boston, MA, USA: NEAVS. Available at: http://www.releasechimps.org/flawed-science/dangerous-and-unnecessary/the-case-to-end-chimpanzee-research/ (Accessed 23.1.12).

12. Bailey, J. (2008). An assessment of the role of chimpanzees in AIDS vaccine research. ATLA 36, 381-428.

13. Bailey, J. (2009). An examination of chimpanzee use in human cancer research. ATLA 37, 399-416.

14. Bailey, J. (2010). An assessment of chimpanzee use in Hepatitis C research past, present and future: 1 . Validity of the chimpanzee model. ATLA 38, 387-418.

15. Bailey, J. (2010). An assessment of chimpanzee use in Hepatitis C research past, present and future: 2. Alternative replacement methods. ATLA 38, 471-494.

16. Anon. (2011). Use of Non-human Primates (NHPs) in the Discovery and Development of Medicines and Vaccines. 
Global Public Policy Issues, 2pp. Brentford, Middlesex, UK: GlaxoSmithKline. Available at: http://www.gsk.com/ policies/GSK-public-position-on-NHP.pdf (Accessed 23.1.12).

17. Bailey, J. (2011). Lessons from chimpanzee-based research on human disease: The implications of genetic differences. ATLA 39, 527-540.

18. Institute of Medicine and National Research Council (2011). Chimpanzees in Biomedical and Behavioral Research. Assessing the Necessity. 200 pp. Washington, DC, USA: The National Academies Press. Available at: http://www.ncbi.nlm.nih.gov/books/NBK82797/pdf/nap13257.pdf (Accessed 23.1.12). 